\title{
Self-similar gravity currents in porous media: Linear stability of the Barenblatt-Pattle solution revisited
}

\author{
Jochonia S. Mathunjwa*, Andrew J. Hogg \\ Centre of Environmental and Geophysical Flows, School of Mathematics, University of Bristol, University Walk, Bristol BS8 1TW, UK
}

Received 25 November 2004; received in revised form 27 July 2005; accepted 15 September 2005

Available online 15 December 2005

\begin{abstract}
We study the linear stability properties of the Barenblatt-Pattle (B-P) self-similar solutions of the porous medium equation which models flow including viscous and porous media gravity currents. Grundy and McLaughlin [R.E. Grundy, R. McLaughlin, Eigenvalues of the Barenblatt-Pattle similarity solution in nonlinear diffusion, Proc. Roy. Soc. London Ser. A 383 (1982) 89-100] have shown that, in both planar and axisymmetric geometries, the B-P solutions are linearly stable to symmetric perturbations. Using a new technique that eliminates singularities in the linear stability analysis, we extend their result and establish that the axisymmetric B-P solution is linearly stable to asymmetric perturbations. This suggests that the axisymmetric B-P solution provides the intermediate asymptotics of gravity currents that evolve from a wide range of initial distributions including those that are not axisymmetric. We use the connection between the perturbation eigenfunctions and the symmetry transformations of the B-P solution to demonstrate that the leading order rate of decay of the perturbations can be maximised by redefining the volume, time and space variables. We show that, in general, radially symmetric perturbations decay faster than asymmetric perturbations of equal amplitude. These theoretical predictions are confirmed by numerical results.
\end{abstract}

(c) 2005 Elsevier SAS. All rights reserved.

Keywords: Porous medium equation; Shallow-water approximation; Self-similar solution; Linear stability analysis; Intermediate asymptotics

\section{Introduction}

The need to understand flow of fluids through porous media is motivated by the pivotal role it plays in many fields of science and engineering such as agriculture, groundwater hydrology, and petroleum engineering. Mathematical studies of porous media flows date back to the nineteenth century. In particular, the flow of fluid through saturated porous media is well established (e.g. Bear [1], Dullien [2]) and the steady-state flow through isotropic homogeneous porous media is governed by Laplace's equation. For unsteady flows, the motion is governed by a coupled system of equations involving the field variables and the position of the moving interface. The system is nonlinear because the position of the interface is not known beforehand, and is determined as part of the solution. This means that, in general, analytical solution is difficult if not impossible. Different approaches have been used to derive limits in which analytical solution of the system is possible. One of the most successful and extensively used of these is the

\footnotetext{
* Corresponding author.

E-mail address: jsmaths@yahoo.co.uk (J.S. Mathunjwa).
} 
shallow-water approximation (see, for example, Bear [1], Huppert and Woods [3], Barenblatt [4], Fowler [5]). Under this approximation, the horizontal length scale of the flow is assumed to be much larger than the vertical length scale, which amounts to assuming that the flow is essentially horizontal with a hydrostatic pressure distribution. The field variables then become functions of the horizontal space coordinates and time only, and the position of the interface can be decoupled from them. For flows where the motion is driven by the gravitational force due to density gradients between the fluids, the position of the interface then satisfies a nonlinear diffusion equation called the porous medium equation (PME). For a gravity current caused by the finite release of a dense fluid along an infinite horizontal boundary in a porous medium saturated with a less dense fluid, a self-similar solution of the PME has been found by Barenblatt [6]. This solution had been identified earlier, in the context of concentration-dependent diffusion by Zel'dovich and Kompaneets [7], and later by Pattle [8], and is widely known as the Barenblatt-Pattle (B-P) solution. The analytical predictions provided by the B-P solution have been shown to be in excellent agreement with experimental results (see, for example, Huppert [9]). Important examples where this model is applicable include the dispersal of pollutants through groundwater subsequent to contamination and the spreading of chemical-laden water injected to sweep oil through the reservoir, after the injection period.

Analytical self-similar solutions are useful tools for scientists and engineers. They provide both significant insight into the dynamical balances that govern the evolution of physical systems and a standard against which experimental and numerical results may be tested. They also provide the asymptotics of classes of physical systems in the intermediate regime when the systems are independent of their initial conditions but have not reached their final equilibrium states. These functions served by self-similar solutions require that they be stable in the sense that the systems evolve towards the self-similar state from arbitrary conditions.

Grundy and McLaughlin [10] (referred to as GM hereafter) have shown that the B-P self-similar solutions in two-dimensional and axisymmetric geometries are linearly stable to disturbances (perturbations) that possess twodimensional and radial symmetry, respectively. They demonstrated that in both geometries small perturbations imposed on the self-similar solution decay in time. Witelski and Bernoff [11] have also studied the two-dimensional problem and obtained similar results to GM. King [12] constructed exact solutions of the multidimensional PME in the form of quadratic polynomials in terms of Cartesian coordinates. Solutions that have bounded spatial domains are shown to decay to the radially symmetric B-P solution at large times, and the rates of decay are determined by utilising integral moments of the motion that remain constant throughout the evolution.

Other studies involving linear stability analyses of self-similar solutions of nonlinear diffusion equations include the following: Halfar ([13] and [14]) has shown the linear stability of self-similar solutions of an equation which describes the evolution of ice sheets. Bernoff and Witelski [15] have shown the linear stability of a self-similar solution of the generalised lubrication equation which models the flow of thin viscous films dominated by surface-tension. Chertock [16] has shown the linear stability of the self-similar solution of an equation which describes the motion of gravity currents that flow through a fissurised porous medium. These nonlinear diffusion equations are usually degenerate at the leading edges where the dependent variable vanishes and the corresponding self-similar solutions are generally characterised by infinite gradients there. This property of the self-similar solutions usually leads to singular terms in the linear stability calculations. GM and Halfar resolved the singularities by using the method of strained coordinates while Witelski and Bernoff [11] transformed the governing equations to self-adjoint form choosing the weight function to absorb the singularities.

In this paper, we introduce a new approach which circumvents these problems by transforming the dependent variable so that the slope of the self-similar solution remains finite at the edges and hence no singularities arise in the linear stability calculations. We extend the analysis of GM by considering the general case where the perturbations imposed on the axisymmetric B-P solution are not necessarily radially symmetric. We show that the self-similar solution is linearly stable to perturbations that depend on both the radial and azimuthal coordinates. This result supports the assertion that the axisymmetric B-P solution provides the intermediate asymptotics of gravity currents evolving from a wide range of initial conditions including those that are asymmetric. By appealing to the connection between the disturbance eigenfunctions and the symmetry transformations of the self-similar solution, we demonstrate that the leading order rate of decay of the disturbance can always be maximised by redefining the volume, time and space variables. The variables in terms of which the rate of decay is maximal are called optimal variables (e.g. Witelski and Bernoff [11]). We show that, both in terms of primitive and optimal variables, volume-preserving disturbances that are axisymmetric in shape typically decay faster than asymmetric disturbances of equal amplitude. The rates of convergence to the self-similar states, which we derive for currents evolving from profiles of arbitrary shapes, reduce 
to the rates obtained by GM in the absence of azimuthal dependence, and are in accord with those found by King [12] for certain exact solutions.

The paper also contains results of the development of gravity currents obtained by numerically integrating from arbitrary initial profiles. The results confirm that currents evolving from arbitrary initial profiles that are sufficiently localised approach the axisymmetric B-P solution in the intermediate regime. Moreover, currents evolving from asymmetric initial profiles are shown to take longer to adjust to self-similar state than those evolving from axisymmetric profiles. The numerically determined rates of convergence to self-similar state are found to be in excellent agreement with those predicted by the linear theory.

The paper is organised as follows: we formulate the problem and present the B-P solution in Section 2. Then, in Section 3, we analyse the evolution of the B-P solution subsequent to the introduction of an asymmetric perturbation. In Section 3.1, we perform a linear stability analysis and derive leading order expressions for the perturbation quantities. The connection between the perturbation eigenfunctions and the symmetry transformations of the B-P solution is discussed in Section 3.2. We present numerical results and compare with theoretical predictions in Section 3.3, after which we give concluding remarks in Section 4. The paper also contains two appendices: Appendix A gives details of the numerical methods that have been used, and Appendix B contains a theoretical derivation of expressions that describe the evolution of the perturbations.

\section{Formulation and self-similar solution}

We study the motion of a radially symmetric gravity current produced by the finite release of fluid of density $\rho+\Delta \rho$ in a porous medium which is saturated with fluid of density $\rho$ over an impermeable horizontal boundary. We employ cylindrical polar coordinates $r, \theta, z$ defined as shown in Fig. 1. We assume that the properties of the porous medium are homogeneous in horizontal directions and, following Huppert and Woods [3], take the porosity to vary linearly in the vertical direction according to

$$
\phi(z)=\phi_{0}+\phi_{1} z / h_{0},
$$

where $h_{0}$ is the typical initial height of the current, and $\phi_{0}>0$ and $\phi_{1}$ are constants. We adopt a power law relationship between permeability and porosity, given by

$$
K(\phi)=K_{0} \phi^{2 \alpha},
$$

where $K_{0}>0$ and $\alpha>0$ are constants. Empirical formulas of this form are used commonly for natural soils and rocks and, for these porous media, the typical value of the exponent falls in the range $1 \leqslant \alpha \leqslant 5$ (Dullien [2], Fowler [5]).

The low Reynolds number flow of fluid through the porous medium is then governed by Darcy's law and the continuity equation:

$$
\begin{aligned}
& \boldsymbol{u}=(u, w)=-\frac{K(z)}{\mu}\{\nabla p+\rho g \hat{\boldsymbol{k}}\}, \\
& \nabla \cdot \boldsymbol{u}=\frac{1}{r} \partial_{r}(r u)+\partial_{z} w=0,
\end{aligned}
$$

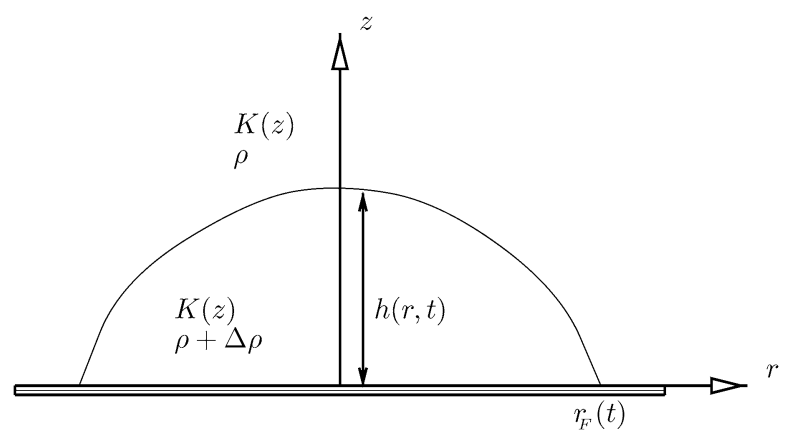

Fig. 1. Definition diagram for a gravity current flowing along a horizontal boundary through a porous medium. 
where $u$ and $w$ are the $r$ - and $z$-components of the transport velocity, respectively, $\mu$ is the dynamic viscosity, $p(r, z, t)$ is the fluid pressure, $g$ is the gravitational acceleration, $\partial_{r} \equiv \partial / \partial r$, and $\hat{\boldsymbol{k}}$ is the unit normal in the vertical direction.

The boundary conditions are

$$
\begin{aligned}
& \phi(h) \partial_{t} h+u \partial_{r} h=w, \quad \text { at } z=h, \\
& \partial_{r} h=0, \quad \text { at } r=0,
\end{aligned}
$$

where (2.5) represents the kinematic condition at the interface and (2.6) is the symmetry condition. At $z=0$, we have zero normal flux, $w=0$, and vanishing height, $h\left(r_{F}, t\right)=0$, where $r_{F}$ is the position of the front.

We further assume that the vertical lengthscale of the current is much smaller than both its horizontal lengthscale and the depth of the ambient fluid. This is the primary assumption of the shallow-water approximation, a technique which has been used extensively to study flows in porous media, and shown to be remarkably effective (see, for example, Bear [1], Huppert and Woods [3], Pritchard et al. [17]). Scaling arguments then indicate that, to leading order, the motion of the current is horizontal and the pressure is hydrostatic, given by

$$
p(r, z, t)=p_{H}+\Delta \rho g(h-z)+\rho g\left(H_{S}-z\right), \quad 0<z<h,
$$

where the constant $p_{H}$ is the pressure at the surface $z=H_{S}$ of the ambient fluid. The evolution equation for the height of the current can now be derived by expressing the kinematic condition (2.5) in terms of $h$ using (2.7), the $r$-component of (2.3), (2.4), and it is given by

$$
(1+\mathcal{K} h) \partial_{t} h=\frac{\mathcal{R}}{r} \partial_{r}\left\{\left[(1+\mathcal{K} h)^{2 \alpha+1}-1\right] r \partial_{r} h\right\},
$$

where $\mathcal{K}=\phi_{1} /\left(\phi_{0} h_{0}\right)$ and $\mathcal{R}=\left(K_{0} \Delta \rho g h_{0} \phi_{0}^{2 \alpha}\right) /\left[(2 \alpha+1) \phi_{1} \mu\right]$. This equation is to be solved subject to the following boundary conditions

$$
\begin{aligned}
& h\left(r_{F}, t\right)=0, \\
& \partial_{r} h(0, t)=0 .
\end{aligned}
$$

Global conservation of volume indicates that

$$
\phi_{0} \int_{0}^{r_{F}} r\left(2 h+\mathcal{K} h^{2}\right) \mathrm{d} r=2 \widetilde{V},
$$

where $2 \pi \tilde{V}$ is the total volume of fluid in the current.

The limit $\phi_{1} / \phi_{0} \rightarrow 0$ corresponds to the case of a porous medium with constant permeability and porosity and has been studied extensively (see, for example, Bear [1], Huppert and Woods [3]). The height of the current in this case satisfies the equation

$$
\partial_{t} h=\mathcal{R}_{0}\left(\partial_{r} \partial_{r}+\frac{1}{r} \partial_{r}\right)\left(\frac{1}{2} h^{2}\right),
$$

where $\mathcal{R}_{0}=K_{0} \phi_{0}^{2 \alpha-1} \Delta \rho g$. This equation is due to Boussinesq [18].

If $\phi_{1} / \phi_{0} \gg 1$, which corresponds to highly variable permeability and porosity, the system of equations (2.8)-(2.11) takes the following approximate form

$$
\begin{aligned}
& h_{0}^{2 \alpha-1} \partial_{t}\left(h^{2}\right)=\mathcal{R}_{1}\left(\partial_{r} \partial_{r}+\frac{1}{r} \partial_{r}\right)\left(\frac{h^{2 \alpha+2}}{\alpha+1}\right), \\
& h\left(r_{F}, t\right)=0, \\
& \partial_{r} h(0, t)=0, \\
& \phi_{1} \int_{0}^{r_{F}} r h^{2} \mathrm{~d} r=2 \widetilde{V} h_{0},
\end{aligned}
$$


where $\mathcal{R}_{1}=\left(K_{0} \Delta \rho g \phi_{1}^{2 \alpha-1}\right) /[(2 \alpha+1) \mu]$ and it has been assumed that $\mathcal{K} h \gg 1$. We nondimensionalise lengths with $h_{0}$ and times with $h_{0} / \mathcal{R}_{1}$, and rescale the height according to $h^{2}=\mathcal{H}$. The rescaled height satisfies the following equation

$$
\partial_{t} \mathcal{H}=\left(\partial_{r} \partial_{r}+\frac{1}{r} \partial_{r}\right)\left(\frac{\mathcal{H}^{\alpha+1}}{\alpha+1}\right)
$$

with the boundary conditions

$$
\begin{aligned}
& \mathcal{H}\left(r_{F}, t\right)=0, \\
& \partial_{r} \mathcal{H}(0, t)=0, \\
& \int_{0}^{r_{F}} r \mathcal{H} \mathrm{d} r=V,
\end{aligned}
$$

where the dimensionless volume $V=2 \widetilde{V} /\left(\phi_{1} h_{0}^{3}\right)$. Eq. (2.17) is a degenerate nonlinear diffusion equation called the porous medium equation (PME) because it was first derived in the study of percolation of gases through porous media (Muskat [19]). ${ }^{1}$ It is degenerate because the coefficient of the highest derivative $\left(\mathcal{H}^{\alpha}\right)$ goes to zero at the edges and hence, while it is of second-order parabolic type in $0 \leqslant r<r_{F}$, Eq. (2.17) degenerates to first order at $r=r_{F}$. We remark that the PME has the same structure as the (Boussinesq) equation (2.12) and setting $\alpha=1$ in (2.17) reduces it into an equation of the same form as Eq. (2.12) governing the flow of fluid through a porous medium of constant permeability.

The PME has also been derived in a number of other applications from different fields including the following: the case $\alpha=3$ describes the height of a viscous free-surface gravity current which propagates on a solid boundary (e.g. Huppert [9]). In high temperature physics, thermal conduction in plasmas is characterised by $\alpha=\frac{5}{2}$ (Zel'dovich and Raizer [20]) and the radiative transfer of heat by Marshak waves during the initial stages of an intense explosion is described by $\alpha=6$ (Larsen and Pomraning [21]), where in these two examples $\mathcal{H}$ denotes temperature. In population dynamics, the evolution of the population of a species in space can also be modelled by (2.17), where $\mathcal{H}$ represents the population density (Murray [22], Witelski [23]). More examples of the application of the porous medium equation are described in, for example, Peletier [24] and Aronson [25]. In this paper, we study the linear stability properties of the self-similar solution of PME.

The problem described by the system (2.17)-(2.20) admits a self-similar solution given by (Barenblatt [4])

$$
\mathcal{H}_{S}(r, t)=t^{-1 /(\alpha+1)} \Psi(\xi)
$$

where

$$
\Psi(\xi)=\left[\frac{\alpha}{4(\alpha+1)}\left(\xi_{F 0}^{2}-\xi^{2}\right)_{+}\right]^{1 / \alpha},
$$

the similarity variable $\xi=r / t^{1 /(2 \alpha+2)}, \xi_{+} \equiv \max (\xi, 0)$ and the position of the front is given by $\xi_{F 0}^{2 \alpha+2}=\{4(1+$ $\left.\left.\frac{1}{\alpha}\right)\right\}^{\alpha+1}\left(\frac{1}{2} V\right)^{\alpha}$. The predictions of this solution have been found to be in excellent agreement with experimental results (see, for example, Huppert [9] for the case $\alpha=3$ ). This confirms the assertion that the self-similar solution, even though singular at $t=0$, describes the intermediate asymptotic behaviour of systems evolving from a wide range of initial conditions. Thus flows with the same volume but different initial distributions evolve towards the same profile given by (2.21) in the intermediate regime. The flows are said to have "forgotten" their initial conditions in this regime.

GM have shown that this solution is linearly stable to radially symmetric disturbances. In their analysis, which involved dealing with singular terms at the front, they found an eigenfunction expansion of the disturbance function in terms of hypergeometric functions and demonstrated that the disturbance decays in time. We extend the analysis of GM to the case of a disturbance which depends on both the radial and azimuthal coordinates. We avoid singularities associated with infinite gradients at the front by introducing an approach in which the problem is transformed into a system of dependent variables for which the gradient of the height remains finite at the front.

\footnotetext{
1 The equation as derived in this context models the two-dimensional adiabatic flow of a gas through a porous medium of constant permeability. The exponent $\alpha$ is a function of the heat capacity ratio $\gamma=C_{P} / C_{V}$, where $C_{P}$ is the specific heat of the gas at constant pressure and $C_{V}$ is the specific heat of the gas at constant volume.
} 


\section{Perturbation of self-similar solution}

We study the development of the solution (2.21) subsequent to the introduction, at some reference time $t_{0}$, say, of a disturbance that depends on both $r$ and $\theta$. For times $t>t_{0}$, derivatives with respect to $\theta$ are no longer identically equal to zero and the governing equation (2.17) is replaced by

$$
\partial_{t} \mathcal{H}=\nabla_{r \theta}^{2}\left(\frac{\mathcal{H}^{\alpha+1}}{\alpha+1}\right),
$$

where $\nabla_{r \theta}^{2}=\partial_{r} \partial_{r}+r^{-1} \partial_{r}+r^{-2} \partial_{\theta} \partial_{\theta}$. The boundary conditions are now given by

$$
\begin{aligned}
& \int_{0}^{2 \pi} \int_{0}^{r_{F}} r \mathcal{H}(r, \theta, t) \mathrm{d} r \mathrm{~d} \theta=2 \pi V, \\
& \mathcal{H}\left(r_{F}, \theta, t\right)=0,
\end{aligned}
$$

where $r_{F}=r_{F}(\theta, t)$. At the origin, the disturbed distribution is not necessarily symmetric and the condition $\partial_{r} \mathcal{H}=0$ is replaced by the requirement that $\mathcal{H}(r, \theta, t)$ must remain finite.

\subsection{Linear stability analysis}

The gradient of the self-similar solution (2.21) becomes infinite at the front when $\alpha>1$. This property of the selfsimilar solution leads to singular terms in the equations governing the perturbation and GM have remedied this by using the method of strained coordinates. We introduce an approach that scales out the singularities by transforming the dependent variable so that the gradient of the self-similar height at the front is finite for all values of $\alpha$. Thus, we write

$$
\mathcal{H}^{\alpha}=\alpha t^{-\alpha /(\alpha+1)} \Phi(\xi, \theta, t) .
$$

While this type of transformation has been used in several studies of the PME (e.g. Gilchrist [26]), we are not aware of work in which it has been used in the context of linear stability analysis.

Substituting (3.4) into (3.1) gives an equation which describes the evolution of $\Phi$

$$
t \partial_{t} \Phi=\alpha \Phi \nabla_{\xi \theta}^{2} \Phi+\left(\nabla_{\xi \theta} \Phi\right)^{2}+\left(\frac{1}{2} \xi \partial_{\xi} \Phi+\alpha \Phi\right) /(\alpha+1) .
$$

We look for a solution of the perturbed problem, $\mathcal{H}_{P}$, by expanding $\Phi$ and $\xi_{F}$ about the self-similar quantities as follows:

$$
\begin{aligned}
& \Phi(\xi, \theta, t)=\Phi_{0}(\xi)+\delta \Phi_{1}(\xi, \theta, t)+\cdots, \\
& \xi_{F}(\theta, t)=\xi_{F 0}+\delta \xi_{F 1}(\theta, t)+\cdots,
\end{aligned}
$$

where $\delta \ll 1$ is the amplitude of the disturbance. Substituting these expressions into (3.5) and comparing powers of $\delta$ yields, at $\mathrm{O}(1)$,

$$
\begin{aligned}
0 & =\alpha \Phi_{0} \Phi_{0}^{\prime \prime}+\alpha \Phi_{0} \Phi_{0}^{\prime} / \xi+\Phi_{0}^{\prime 2}+\left(\frac{1}{2} \xi \Phi_{0}^{\prime}+\alpha \Phi_{0}\right) /(\alpha+1), \\
& =\frac{1}{\xi}\left(\alpha \Phi_{0} \frac{\mathrm{d}}{\mathrm{d} \xi}+\Phi_{0}^{\prime}\right)\left(\xi \Phi_{0}^{\prime}+\frac{\xi^{2}}{2(\alpha+1)}\right),
\end{aligned}
$$

where prime denotes differentiation with respect to $\xi$. The boundary conditions are

$$
\begin{aligned}
& \int_{0}^{\xi_{F 0}} \xi\left(\alpha \Phi_{0}\right)^{1 / \alpha} \mathrm{d} \xi=V, \\
& \Phi_{0}^{\prime}(0)=0, \\
& \Phi_{0}\left(\xi_{F 0}\right)=0 .
\end{aligned}
$$


Solving the system (3.8)-(3.11) recovers the self-similar solution, given by

$$
\Phi_{0}(\xi)=\frac{1}{4(\alpha+1)}\left(\xi_{F 0}^{2}-\xi^{2}\right) .
$$

At $O(\delta)$, the equation governing the development of $\Phi_{1}$ is found to be

$$
4\left(1+\frac{1}{\alpha}\right) t \partial_{t} \Phi_{1}=\left(\xi_{F 0}^{2}-\xi^{2}\right) \nabla_{\xi \theta}^{2} \Phi_{1}-\frac{2}{\alpha} \xi \partial_{\xi} \Phi_{1},
$$

where the expression (3.12) has been substituted for $\Phi_{0}$. The boundary conditions are given by

$$
\begin{aligned}
& \int_{0}^{2 \pi} \int_{0}^{\xi_{F 0}} \xi \Phi_{0}^{1 / \alpha-1} \Phi_{1} \mathrm{~d} \xi \mathrm{d} \theta=0, \\
& \Phi_{1}\left(\xi_{F 0}, \theta, t\right)=\frac{1}{2} \xi_{F 0} \xi_{F 1} /(\alpha+1),
\end{aligned}
$$

and the requirement that the function $\Phi_{1}$ must remain finite at the origin. The condition (3.14) expresses volume conservation for volume-preserving perturbations and (3.15) expresses the variation in the position of the front caused by the perturbations.

It is convenient to introduce the transformations $\eta=\xi / \xi_{F 0}$ and $\tau=\ln \left(t / t_{0}\right)$, in terms of which the time at which the self-similar solution is disturbed becomes $\tau=0$ and the position of the front is $\eta=1$, to leading order. The governing equation (3.13) becomes

$$
4\left(1+\frac{1}{\alpha}\right) \partial_{\tau} \Phi_{1}=\left(1-\eta^{2}\right) \nabla_{\eta \theta}^{2} \Phi_{1}-\frac{2}{\alpha} \eta \partial_{\eta} \Phi_{1}
$$

Since this is a linear homogeneous equation, we use the method of separation of variables and look for a solution in separable form

$$
\begin{aligned}
& \Phi_{1}(\eta, \theta, \tau)=\sum_{n=0}^{\infty} \sum_{m=0}^{\infty} a_{m n} \mathrm{e}^{\mathrm{i} m \theta-\lambda_{m n} \tau} \mathcal{F}_{m n}\left(\eta ; \lambda_{m n}\right), \\
& \eta_{F 1}(\theta, \tau)=\sum_{n=0}^{\infty} \sum_{m=0}^{\infty} b_{m n} \mathrm{e}^{\mathrm{i} m \theta-\lambda_{m n} \tau},
\end{aligned}
$$

where $\mathrm{i}=\sqrt{-1}, a_{m n}$ and $b_{m n}$ are constants, and $\lambda_{m n}$ are the eigenvalues of the problem. Thus, establishing linear stability of the self-similar solution (2.21) involves showing that none of the eigenvalues $\lambda_{m n}$ are negative. The eigenfunctions $\mathcal{F}_{m n}$ satisfy the ordinary differential equation

$$
\eta^{2}\left(1-\eta^{2}\right) \mathcal{F}_{m n}^{\prime \prime}+\eta\left\{1-\left(1+\frac{2}{\alpha}\right) \eta^{2}\right\} \mathcal{F}_{m n}^{\prime}+\left\{\left[m^{2}+4\left(1+\frac{1}{\alpha}\right) \lambda_{m n}\right] \eta^{2}-m^{2}\right\} \mathcal{F}_{m n}=0
$$

Then, making the substitutions $s=\eta^{2}, \mathcal{F}_{m n}(s)=s^{\frac{1}{2} m} \mathcal{X}_{m n}(s)$ and $2 s=\zeta+1$ in succession transforms the Eq. (3.19) into a Jacobi differential equation (Abramowitz and Stegun [27])

$$
\left(1-\zeta^{2}\right) \mathcal{X}_{m n}^{\prime \prime}+\left\{1+m-\left(1+m+\frac{1}{\alpha}\right) \zeta\right\} \mathcal{X}_{m n}^{\prime}+\left\{\left(1+\frac{1}{\alpha}\right) \lambda_{m n}-\frac{m}{2 \alpha}\right\} \mathcal{X}_{m n}=0
$$

defined in the interval $-1 \leqslant \zeta \leqslant 1$. The end points $\zeta= \pm 1$ are regular singular points of (3.20) and solutions which are continuous in the whole interval exist only when the coefficient multiplying $\mathcal{X}_{m n}$ equals $n(n+a+b+1)$, and are given by

$$
\mathcal{X}_{m n}(\zeta)=P_{n}^{(a, b)}(\zeta)
$$

where $P_{n}^{(a, b)}$ denotes a Jacobi polynomial of degree $n$, and $a=(1-\alpha) / \alpha, b=m$. The first three Jacobi polynomials are given by 


$$
\begin{aligned}
& P_{0}^{(a, b)}(\zeta)=1, \\
& P_{1}^{(a, b)}(\zeta)=\frac{1}{2}(a-b)+\frac{1}{2}(2+a+b) \zeta, \\
& P_{2}^{(a, b)}(\zeta)=\frac{1}{8}\left[(a-b)^{2}-(4+a+b)\right]+\frac{1}{8}(3+a+b)\left[2(a-b) \zeta+(4+a+b) \zeta^{2}\right] .
\end{aligned}
$$

Higher degree polynomials can then be calculated using the recurrence relation

$$
2 \mathcal{B}(n+1)(\mathcal{B}-n+1) P_{n+1}=\left\{(\mathcal{B}+1)\left(a^{2}-b^{2}\right)+(\mathcal{B})_{3} \zeta\right\} P_{n}-2(n+a)(n+b)(\mathcal{B}+2) P_{n-1},
$$

where $P_{n} \equiv P_{n}^{(a, b)}(\zeta), \mathcal{B}=2 n+a+b$ and the Pochhammer symbol $(m)_{n}=(m-n+1) ! /(m-1) !$. An important feature of the Jacobi polynomials is that they form a complete set of orthogonal functions in $-1 \leqslant \zeta \leqslant 1$ with respect to the weight function $(1-\zeta)^{a}(1+\zeta)^{b}$. This implies that any piecewise continuous function in $-1 \leqslant \zeta \leqslant 1$ can be represented by a unique series of Jacobi polynomials.

By demanding that the solution exist throughout $-1 \leqslant \zeta \leqslant 1$, we deduce that the eigenvalues are given by

$$
\lambda_{m n}=\frac{1}{\alpha+1}\left\{\alpha n(n+m)+n+\frac{1}{2} m\right\}, \quad n, m=0,1,2, \ldots
$$

This formula indicates that small $\theta$-dependent disturbances imposed on the self-similar solution (2.21) decay in time because all eigenvalues $\lambda_{m n}$ are nonnegative, and hence (2.21) is linearly stable to asymmetric disturbances. The eigenvalue $\lambda_{00}=0$ is associated with symmetry transformations of the problem which alter the volume of the current and we demonstrate below that it does not negate the stability.

Setting $m=0$ in (3.22) recovers the formula obtained by GM for radially symmetric disturbances, namely

$$
\lambda_{0 n}=\frac{n(\alpha n+1)}{\alpha+1}, \quad n=0,1,2, \ldots
$$

The eigenfunction associated with $\lambda_{m n}$ is then given by

$$
\mathcal{F}_{m n}(\eta)=\eta^{m} P_{n}^{(a, b)}\left(2 \eta^{2}-1\right), \quad a=\frac{1}{\alpha}-1, b=m,
$$

and its form, for various values of $m$ and $n$, is illustrated in Fig. 2. The conservation of volume integral (3.14) is automatically satisfied by all eigenfunctions except $\mathcal{F}_{00}$. Applying the condition (3.15) at the front yields

$$
b_{m n}=2 a_{m n}(\alpha+1) P_{n}^{(a, b)}(1) / \xi_{F 0}^{2} .
$$

At the origin, while the eigenfunctions are not necessarily symmetric, as expected, they all satisfy the requirement of being finite. The perturbation quantities are therefore given by

$$
\begin{aligned}
& \Phi_{1}(\eta, \theta, t)=\sum_{m=0}^{\infty} \sum_{n=0}^{\infty} a_{m n}\left(\frac{t_{0}}{t}\right)^{\lambda_{m n}} \mathrm{e}^{\mathrm{i} m \theta} \mathcal{F}_{m n}(\eta), \\
& \eta_{F 1}(\theta, t)=2(\alpha+1) \sum_{m=0}^{\infty} \sum_{n=0}^{\infty} a_{m n} P_{n}^{(a, b)}(1)\left(\frac{t_{0}}{t}\right)^{\lambda_{m n}} \mathrm{e}^{\mathrm{i} m \theta} / \xi_{F 0}^{2},
\end{aligned}
$$

and the perturbed height is given by

$$
\mathcal{H}_{P}(\eta, \theta, t)=t^{-1 /(\alpha+1)}\left\{\mathcal{G}^{1 / \alpha}+\frac{\delta \mathcal{G}^{1 / \alpha-1}}{\alpha} \sum_{m=0}^{\infty} \sum_{n=0}^{\infty} a_{m n}\left(\frac{t_{0}}{t}\right)^{\lambda_{m n}} \mathrm{e}^{\mathrm{i} m \theta} \mathcal{F}_{m n}(\eta)+\mathrm{O}\left(\delta^{2}\right)\right\},
$$

where

$$
\mathcal{G}=\frac{\alpha \xi_{F 0}^{2}}{4(\alpha+1)}\left(1-\eta^{2}\right)
$$

The constants $a_{m n}$ are determined from the initial conditions at $t=t_{0}$ by making use of the orthogonality and completeness properties of the eigenfunctions.

Thus we have established that the axisymmetric B-P solution is linearly stable to asymmetric perturbations without having to resolve singularities associated with infinite gradients at the front of the current. This result supports the 

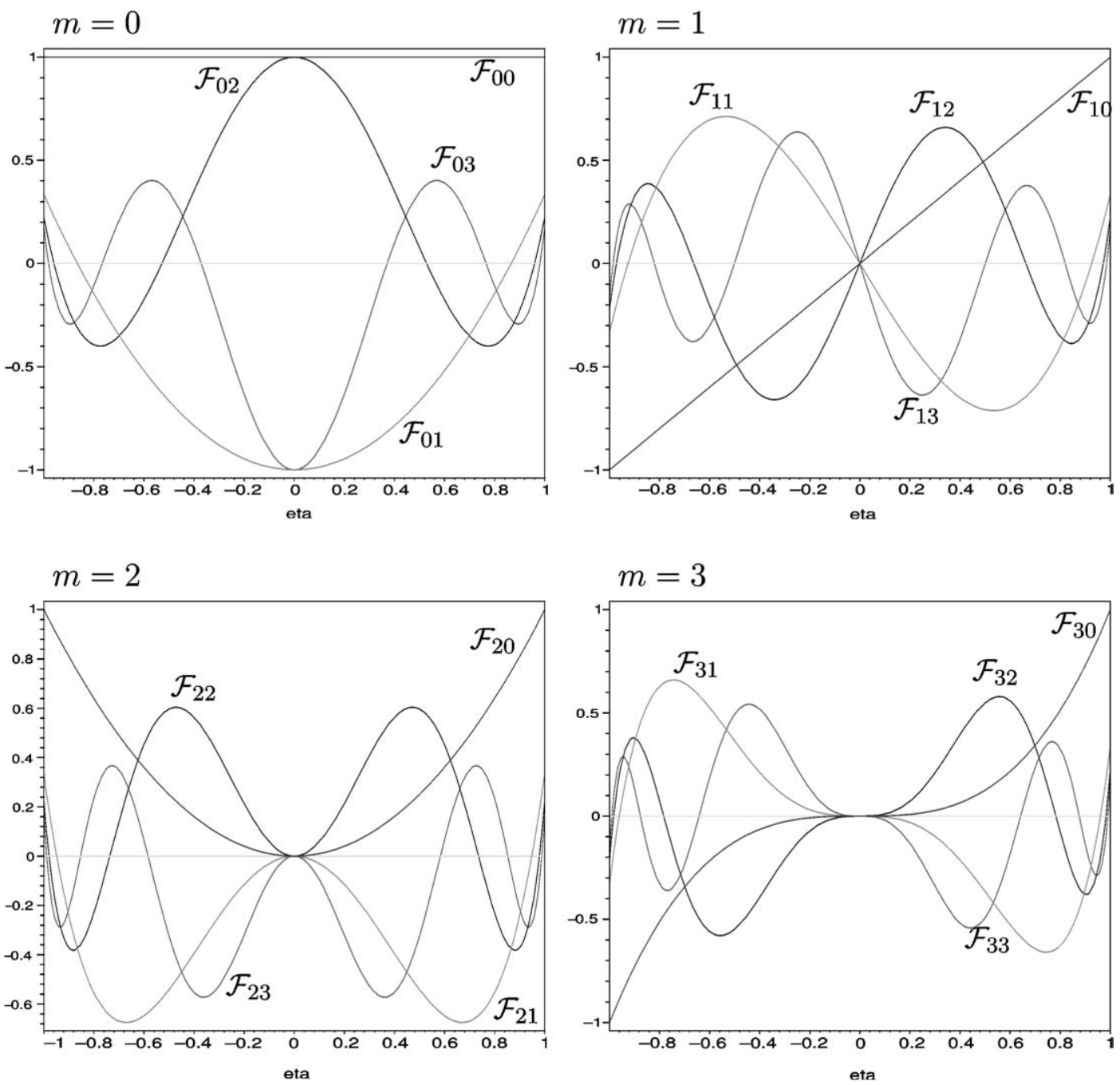

Fig. 2. The first sixteen eigenfunctions of the disturbance function $\Phi_{1}$ plotted as functions of the radial similarity variable $\eta$, calculated for $\alpha=3$. The positive and negative $\eta$-axes represent the $\theta=0$ and $\theta=\pi$ directions, respectively.

assertion that the axisymmetric B-P solution represents the intermediate asymptotic development of currents evolving from a wide variety of initial distributions including those that do not possess axis symmetry. We note that the approach of transforming the dependent variable so that the gradient of the profile at the front remains finite is applicable to other self-similar solutions of nonlinear diffusion equations with infinite gradients at the edges. We have used the approach to study the linear stability of the planar B-P solution to perturbations that possess planar symmetry. We have found a perturbation eigenfunction expansion in terms of Gegenbauer polynomials and all eigenfunctions are shown to decay in time. Hence, the analysis recovers the results presented by GM and Witelski and Bernoff [11] without having to deal with singular terms at the leading edge.

\subsection{Connection with symmetry transformations}

It is convenient to express the self-similar solution in terms of the primitive variables

$$
\mathcal{H}_{S}(r, t)=\left\{\frac{\alpha}{4(\alpha+1)}\left[\frac{\xi_{F 0}^{2}}{t^{\alpha /(\alpha+1)}}-\frac{r^{2}}{t}\right]_{+}\right\}^{1 / \alpha} .
$$


This solution describes the evolution of a gravity current with volume $2 \pi V$ initially concentrated at $r=0$ at time $t=0$. However, these choices of the origin in space and time, and the value of the initial volume are arbitrary and have been made for convenience. We expect the solution of a system of volume $2 \pi \widehat{V}$, initially centered at $r=\hat{r} \mathrm{e}^{\mathrm{i} \theta}$ at time $t=\hat{t}$, to have the same qualitative features as (3.28). In fact, it is straightforward to show that the solution of such a system is also given by (3.28) with $r, t$ and $V$ replaced by $r-\hat{r} \mathrm{e}^{\mathrm{i} \theta}, t-\hat{t}$ and $\widehat{V}$, respectively. This indicates that the self-similar solution (3.28) is invariant under transformations that translate the spatial origin, shift time, or alter the volume. We note that, while the distribution remains axisymmetric under the action of volume-altering and time-shifting transformations, the action of space-translating transformations introduces $\theta$-dependence.

Let $\mathcal{H}_{\sigma}(r, t ; \sigma)$ be the image of the similarity solution $\mathcal{H}_{S}(r, t)$ under the action of a family of point transformations depending on a real continuous parameter $\sigma$. For sufficiently small $\sigma, \mathcal{H}_{\sigma}$ can be approximated by a Taylor series expansion about $\mathcal{H}_{S}$ as

$$
\mathcal{H}_{\sigma}(r, t ; \sigma)=\mathcal{H}_{\sigma}(r, t ; 0)+\left.\sigma \frac{\partial \mathcal{H}_{\sigma}}{\partial \sigma}\right|_{\sigma=0}+\mathrm{O}\left(\sigma^{2}\right),
$$

where $\mathcal{H}_{\sigma}(r, t ; 0)=\mathcal{H}_{S}(r, t)$. This first-order approximation is known as the infinitesimal transformation and the function $\left[\partial \mathcal{H}_{\sigma} / \partial \sigma\right]_{\sigma=0}$ is called the coefficient of the infinitesimal transformation (see, for example, Dresner [28]).

We demonstrate that the eigenfunctions corresponding to $\lambda_{00}=0, \lambda_{01}=1$ and $\lambda_{10}=1 /(2 \alpha+2)$ are equivalent to the coefficients of volume-altering, time-shifting, and space-translating infinitesimal transformations, respectively.

Transformations that alter the volume are defined by $V \mapsto V+\sigma$ and the image of the self-similar solution (3.28) is given by

$$
\begin{aligned}
\mathcal{H}_{\sigma}(r, t ; \sigma) & =\left\{\frac{\alpha}{4(\alpha+1)}\left[4\left(1+\frac{1}{\alpha}\right)\left(\frac{V+\sigma}{2 t}\right)^{\alpha /(\alpha+1)}-\frac{r^{2}}{t}\right]_{+}\right\}^{1 / \alpha}, \\
& =t^{-1 /(\alpha+1)}\left\{\mathcal{G}^{1 / \alpha}+\sigma \mathcal{G}^{1 / \alpha-1} \frac{\alpha \xi_{F 0}^{2}}{4(\alpha+1)^{2} V}+\mathrm{O}\left(\sigma^{2}\right)\right\} .
\end{aligned}
$$

The coefficient of the infinitesimal transformation is a constant and thus equivalent to the $\lambda_{00}$-term in the expansion (3.27).

Transformations that shift time are defined by $t \mapsto t+\sigma$ and the image of (3.28) becomes

$$
\begin{aligned}
\mathcal{H}_{\sigma}(r, t ; \sigma) & =\left\{\frac{\alpha}{4(\alpha+1)}\left[\frac{\xi_{F 0}^{2}}{(t+\sigma)^{\alpha /(\alpha+1)}}-\frac{r^{2}}{t+\sigma}\right]_{+}\right\}^{1 / \alpha}, \\
& =t^{-1 /(\alpha+1)}\left\{\mathcal{G}^{1 / \alpha}+\sigma \mathcal{G}^{1 / \alpha-1} \frac{\alpha \xi_{F 0}^{2}}{(\alpha+1)^{2} t}\left[\left(1+\frac{1}{\alpha}\right) \eta^{2}-1\right]+\mathrm{O}\left(\sigma^{2}\right)\right\} .
\end{aligned}
$$

The coefficient of the infinitesimal transformation is equivalent to the $\lambda_{01}$-term in (3.27).

Fig. 3 shows perturbed profiles produced by adding axisymmetric eigenfunctions $\mathcal{F}_{0 n}$ onto the self-similar profile $\Phi_{0}$. The graphs illustrate that adding the eigenfunction $\mathcal{F}_{00}$ results in a simple vertical translation of the self-similar profile, an effect which is similar to that of an increase in the volume of the current. The effect produced by adding the eigenfunction $\mathcal{F}_{01}$ is a flattening out of the profile, resembling the changes undergone by the profile $\mathcal{H}_{S}(r, t)$ as time evolves.

For radially symmetric disturbances $(m=0)$, there is no eigenfunction associated with space-translating transformations because these transformations introduce $\theta$-dependence. Thus, for these disturbances, it is always possible to redefine the volume and time variable so that $\mathcal{F}_{00}=\mathcal{F}_{01}=0$ in the eigenfunction expansion. The variables in terms of which the disturbance does not have projections onto $\mathcal{F}_{00}$ and $\mathcal{F}_{01}$ are called optimal similarity variables (Witelski and Bernoff [11]). In terms of the optimal variables, therefore, the rate of decay of the disturbance is maximised. The leading order eigenfunction is, then, $\mathcal{F}_{02}$ which decays like $t^{-4+2 /(\alpha+1)}$.

For $\theta$-dependent disturbances, we demonstrate that the first term with $m=1$, corresponding to the eigenvalue $\lambda_{10}=1 /(2 \alpha+2)$, is associated with spatial translations. Space-translating transformations are defined by $r \mapsto r+\sigma \mathrm{e}^{\mathrm{i} \theta}$ and the image of the self-similar solution (3.28) becomes 


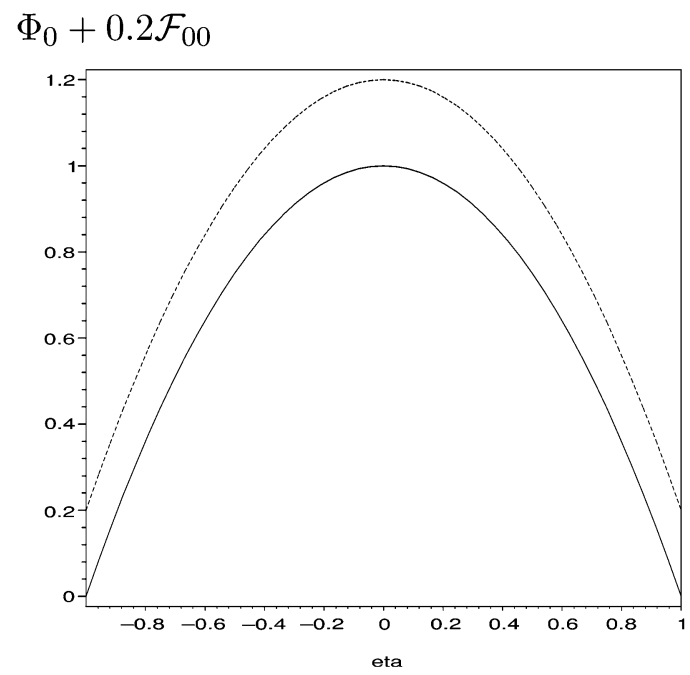

$\Phi_{0}+0.2 \mathcal{F}_{01}$
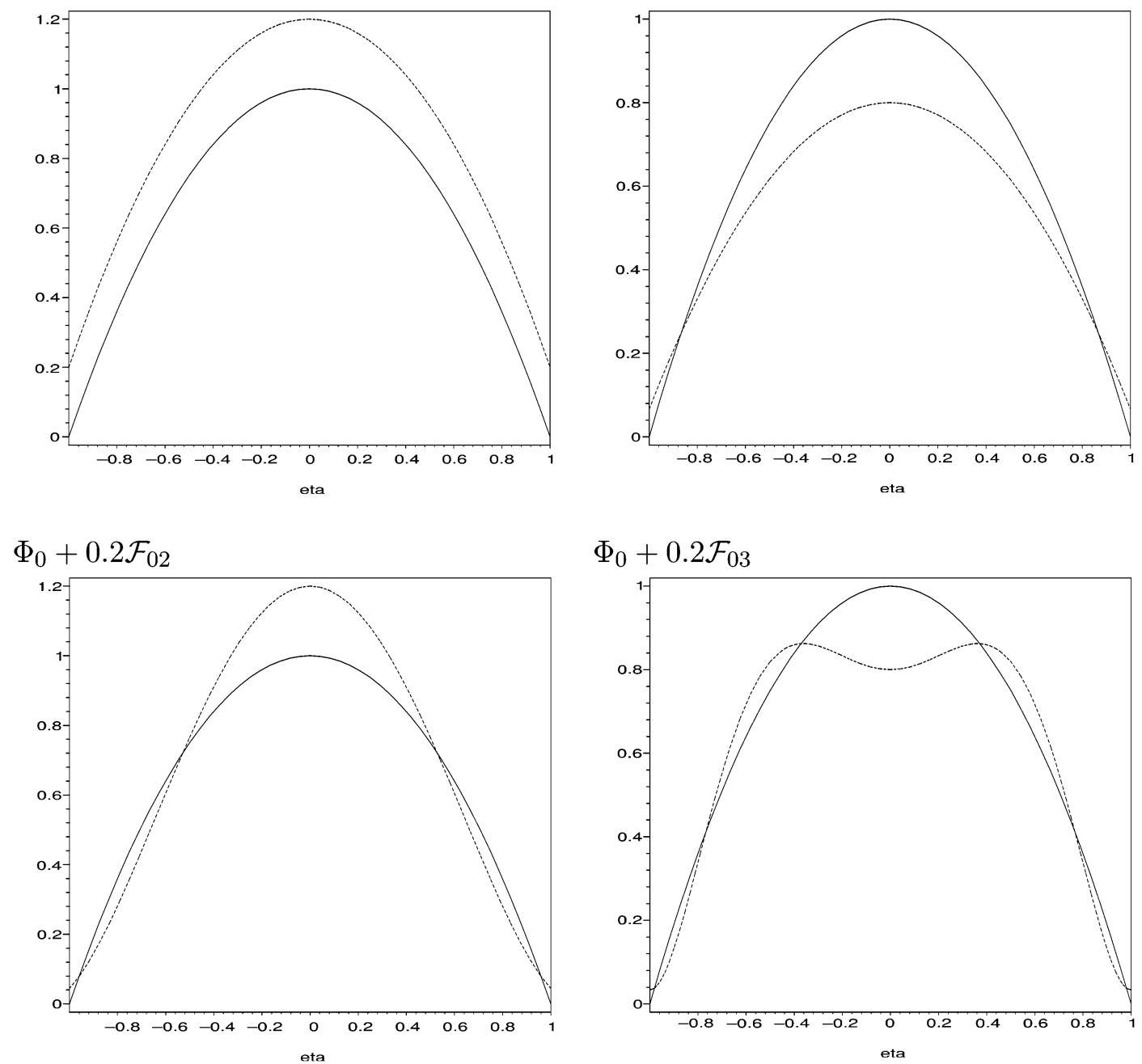

\section{$\Phi_{0}+0.2 \mathcal{F}_{03}$}

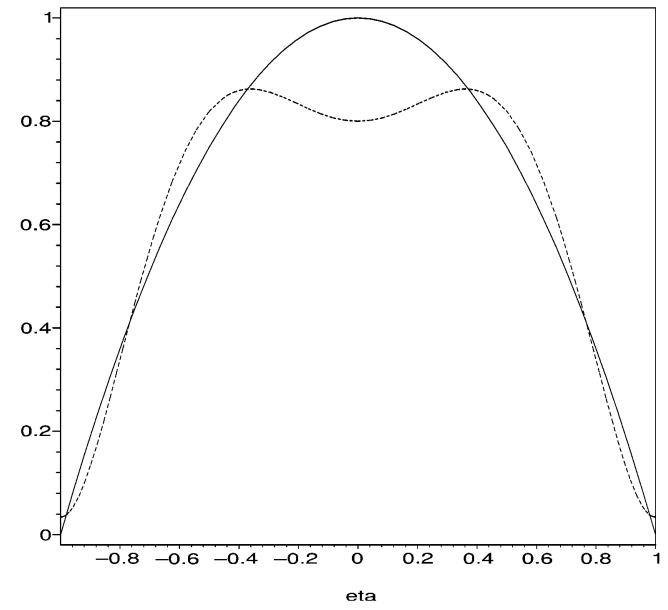

Fig. 3. The self-similar profile $\Phi_{0}$ (solid) and perturbed profiles $\Phi_{0}+0.2 \mathcal{F}_{0 n}$ (dashed) for $n=0,1,2,3$, respectively, plotted as a function of the rescaled similarity variable $\eta$. The positive and negative $\eta$-axes represent the $\theta=0$ and $\theta=\pi$ directions, respectively. In all cases $\alpha=3$.

$$
\begin{aligned}
\mathcal{H}_{\sigma}(r, \theta, t ; \sigma) & =\left\{\frac{\alpha}{4(\alpha+1)}\left[\frac{\xi_{F 0}^{2}}{t^{\alpha /(\alpha+1)}}-\frac{\left(r+\sigma \mathrm{e}^{\mathrm{i} \theta}\right)^{2}}{t}\right]_{+}\right\}^{1 / \alpha} \\
& =t^{-1 /(\alpha+1)}\left\{\mathcal{G}^{1 / \alpha}+\sigma \mathcal{G}^{1 / \alpha-1} \frac{\xi_{F 0}}{2(\alpha+1) V^{\alpha /(\alpha+1)}} \frac{\eta \mathrm{e}^{\mathrm{i} \theta}}{t^{1 /(2(\alpha+1))}}+\mathrm{O}\left(\sigma^{2}\right)\right\} .
\end{aligned}
$$

The coefficient of the infinitesimal transformation is equivalent to the $\lambda_{10}$-term in (3.27). Fig. 4 shows shapes of edges of perturbed currents for $\theta$-dependent perturbations. The plots indicate that the perturbed edge for $m=1$ remains a circle centred at a point different from the origin, which confirms that the $\lambda_{01}$ eigenfunction is equivalent to spacetranslating transformations. Thus, for $\theta$-dependent disturbances, optimal variables can always be defined so that the leading order eigenfunction corresponds to $\lambda_{20}=1 /(\alpha+1)$ and decays like $t^{-1 /(\alpha+1)}$. Since $\alpha>0$, this demonstrates that, in terms of optimal variables, perturbations that depend on both $r$ and $\theta$ imposed on the self-similar solution (2.21) will typically take longer to decay than radially symmetric perturbations of equal amplitude. In fact, even in terms of non-optimal variables, axisymmetric volume-preserving perturbations, which decay like $t^{-1}$, to leading order, will decay faster than $\theta$-dependent perturbations with a leading order rate of decay of $t^{-1 /(2 \alpha+2)}$. We remark that the rates of convergence to self-similar state, here derived for profiles of arbitrary shape, are in accord with those found by King [12] for certain exact solutions of the PME. 

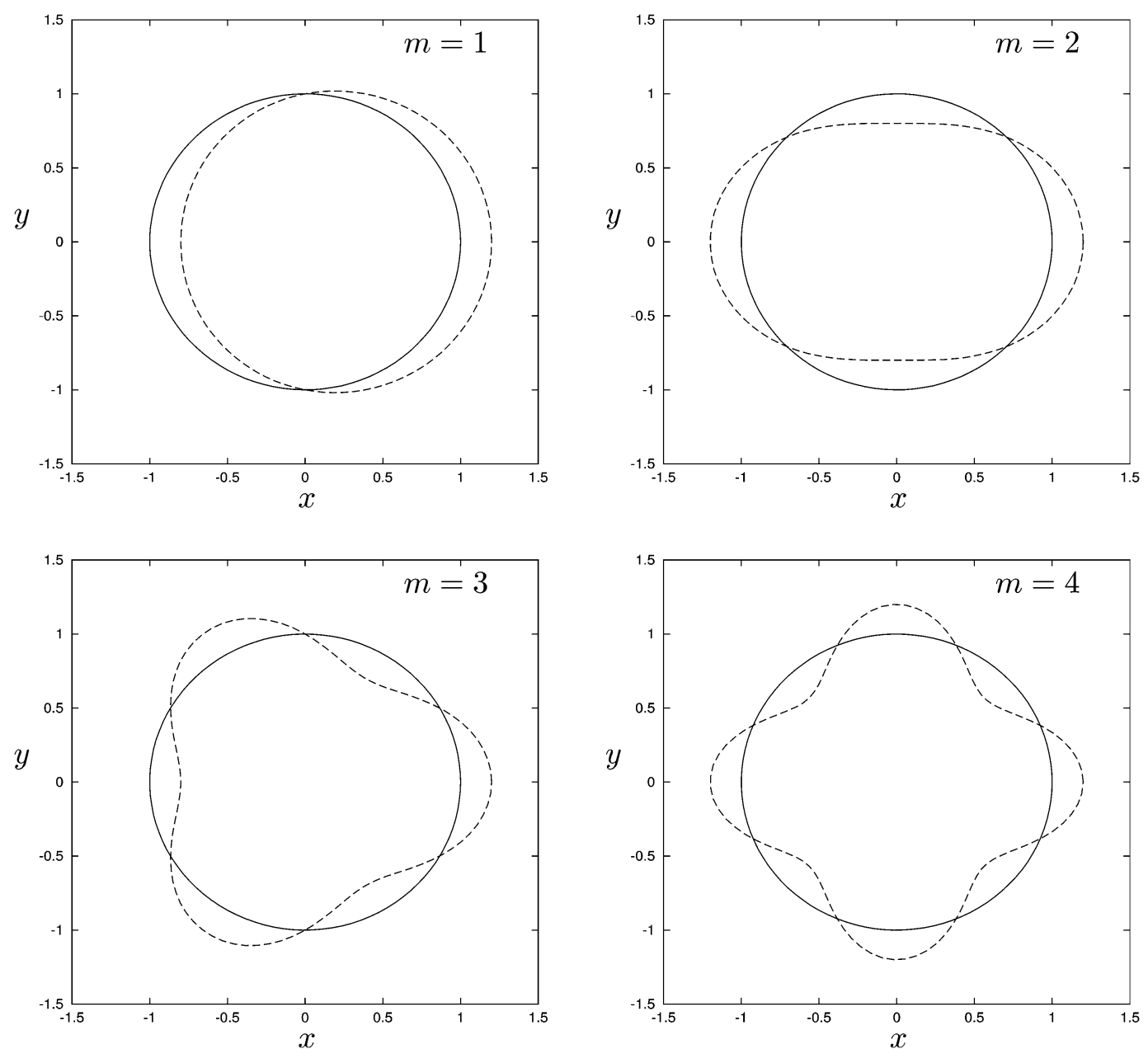

Fig. 4. The edge of the self-similar current $\eta_{F 0}=1$ (solid line) plotted with perturbed edges $1+0.2 \mathrm{e}^{\mathrm{i} m \theta}$ (dashed line) for $m=1,2,3$ and 4. Note that the structure of the perturbed edges is not affected by the values of $\alpha$.

\subsection{Numerical solutions}

We have performed numerical integrations of the governing equation (3.1) from arbitrary axisymmetric and asymmetric distributions to investigate whether axisymmetric solutions converge to the self-similar solution (2.21) more rapidly than asymmetric ones. We also analyse the rates of convergence to self-similar state and compare with theoretical predictions.

To avoid computational difficulties associated with moving boundaries, it is convenient first to transform the problem onto a fixed domain. Such an approach has been proposed by Gurtin et al. [29] and Berryman [30] who employed a Lagrangian frame of reference to integrate the equations. The approach here differs in that we utilise a stretched Eulerian grid. Thus we introduce a stretched radial coordinate $R$ defined by

$$
R=\frac{r}{r_{F}(\theta, t)} .
$$

The governing equation (3.1) is then transformed into

$$
\partial_{t} \mathcal{H}=\frac{R \dot{r}_{F}}{r_{F}} \partial_{R} \mathcal{H}+\frac{1}{r_{F}^{2}} \nabla_{R \theta}^{2}\left(\frac{\mathcal{H}^{\alpha+1}}{\alpha+1}\right)
$$


where

$$
\nabla_{R \theta}^{2}=\left(1+\frac{r_{F}^{\prime 2}}{r_{F}^{2}}\right) \frac{\partial^{2}}{\partial R^{2}}+\left(1+\frac{2 r_{F}^{\prime 2}}{r_{F}^{2}}-\frac{r_{F}^{\prime \prime}}{r_{F}}\right) \frac{1}{R} \frac{\partial}{\partial R}-\frac{2 r_{F}^{\prime}}{r_{F}} \frac{1}{R} \frac{\partial^{2}}{\partial R \partial \theta}+\frac{1}{R^{2}} \frac{\partial^{2}}{\partial \theta^{2}},
$$

$\dot{r}_{F}=\partial_{t} r_{F}$, and $r_{F}^{\prime}=\partial_{\theta} r_{F}$. In terms of the coordinate $R$, the edge of the current is a unit circle at all times and the boundary condition there is

$$
h(R=1, \theta, t)=0 .
$$

For a given height distribution, the evolution of the current is calculated by numerically integrating (3.31) subject to (3.32). The rate of advance of the edge, $\dot{r}_{F}$, is obtained by evaluating (3.31) at $R=1$, and it is given by

$$
\dot{r}_{F}=-\frac{1}{r_{F}}\left(1+\frac{r_{F}^{\prime 2}}{r_{F}^{2}}\right)\left[\partial_{R} \mathcal{H}\right]_{R=1} .
$$

This equation is used iteratively to update the position of the edge during the numerical integrations. Further details of the numerical procedure are presented in Appendix A.

All our numerical calculations have been made for the case $\alpha=1$ although we note that similar calculations can be performed for other values of $\alpha$. The position of the edge of the self-similar distribution is given by $r_{F 0}=(32 V t)^{1 / 4}$. In the $(\Lambda, t)$ frame, where $\Lambda=r /(32 V t)^{1 / 4}$, the edge of the self-similar distribution is invariant in time and it is given by the unit circle $\Lambda=1$. For a current that evolves from an arbitrary non-self-similar profile at $t=T_{0}$, say, the position of the edge in the $(\Lambda, t)$ frame, given by $\Lambda_{F}(\theta, t)=r_{F}(\theta, t) /(32 V t)^{1 / 4}$, will approach the unit circle $\Lambda=1$ as the current adjusts to self-similar state. We introduce a parameter that measures the deviation between $\Lambda_{F}$ and $\Lambda=1$ defined by

$$
\mathcal{D}_{F}(t)=\frac{1}{2 \pi} \int_{0}^{2 \pi}\left|1-\Lambda_{F}(\theta, t)\right| \mathrm{d} \theta
$$

We use this parameter in our numerical calculations to analyse the rate of convergence to self-similar state from different initial distributions. The advantage of the parameter $\mathcal{D}_{F}(t)$, evaluated in the $(\Lambda, t)$ frame, is that it can be used to compare rates of approach to self-similar state of currents even if they have different volumes.

We have carried out integrations from initial distributions of arbitrary shapes. We present results for integrations from two axisymmetric and three asymmetric initial profiles, given by

$$
\begin{aligned}
& \mathcal{H}(r)=\sqrt{\frac{5}{2}}[1-\beta r]_{+}, \\
& \mathcal{H}(r)=\sqrt{\frac{5}{2}} \begin{cases}{\left[5-5^{4 / 5}\right] \beta r,} & 0 \leqslant \beta r<\frac{1}{5}, \\
{\left[1-(\beta r)^{1 / 5}\right],} & \frac{1}{5} \leqslant \beta r \leqslant 1,\end{cases}
\end{aligned}
$$

and

$$
\begin{aligned}
& \mathcal{H}(r, \theta)=\sqrt{\frac{5}{2}}\left\{1-\beta r\left[1+\mathrm{e}^{4 \cos \theta-4}\right]\right\}_{+}, \\
& \mathcal{H}(r, \theta)=\sqrt{\frac{5}{2}}\left\{1-\beta r\left[1+\frac{1}{5} \mathrm{e}^{4 \cos \theta-4}\right]\right\}_{+}, \\
& \mathcal{H}(r, \theta)=\sqrt{\frac{5}{2}}\left\{1-\beta r\left[1+\frac{1}{5} \sin ^{2} \theta\right]\right\}_{+},
\end{aligned}
$$

respectively, where $\beta=\left(\frac{5}{32}\right)^{1 / 4}$. All numerical calculations were started at time $T_{0}=\frac{1}{10}$.

Fig. 5(a) shows the development of the rescaled height profile as a function of $R$ for the current that evolves from (3.36). The height profile is shown to approach the self-similar profile in time. Fig. 5(b) shows the rescaled edge $\Lambda_{F}$ plotted against $\theta$, for the current that evolves from the asymmetric profile (3.37). The plot shows that as time increases the curve of $\Lambda_{F}$ tends to $\Lambda=1$ and thus the leading edge approaches a circular shape (but at a slow rate of 


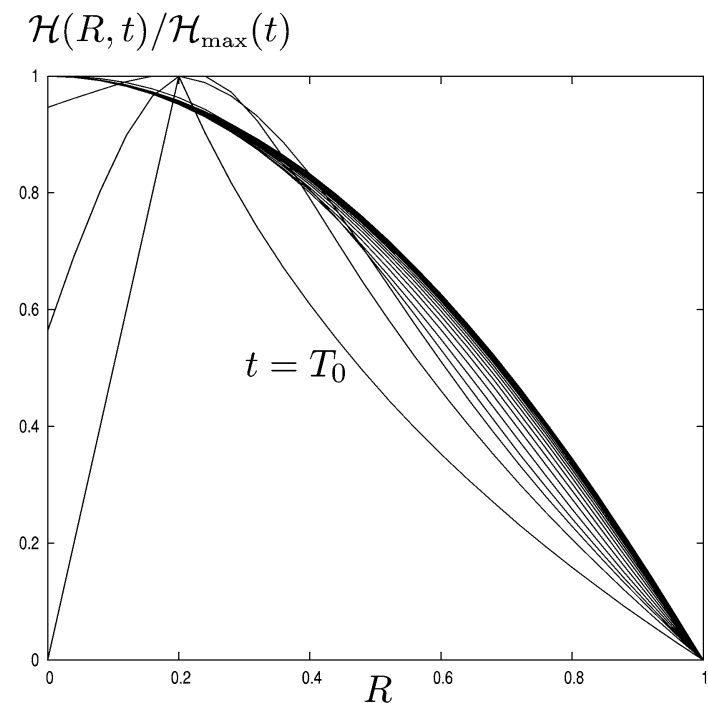

(a)
$\Lambda_{F}(\theta, t)$

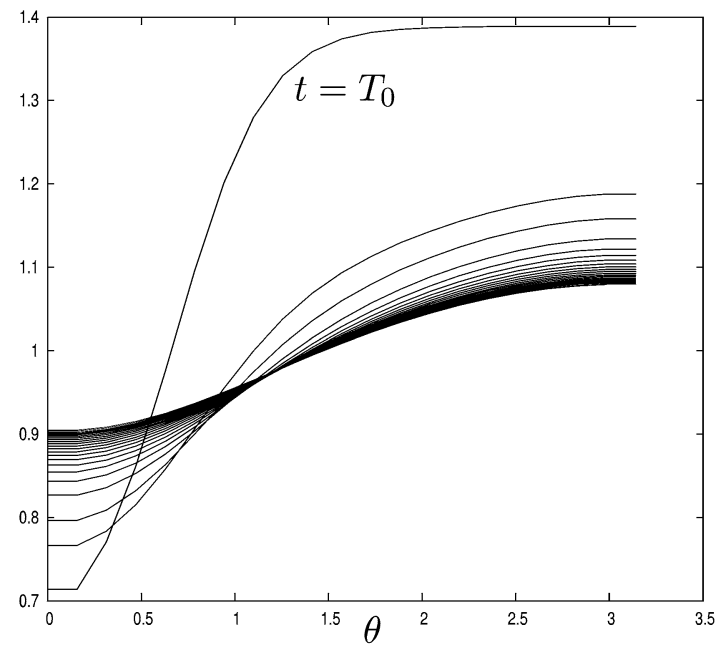

(b)

Fig. 5. (a) The rescaled height profile $\mathcal{H}(R, t) / \mathcal{H}_{\max }(t)$ plotted against $R$ at different times as the current adjusts to self-similar shape from the initial distribution (3.36). (b) The edge $\Lambda_{F}(\theta, t)$ plotted against $\theta$ at different times as the current adjusts to a circular self-similar edge from the asymmetric initial edge of the height profile (3.37), given by $r_{F}(\theta)=\left[\beta+\beta \mathrm{e}^{4(\cos \theta-1)}\right]^{-1}$.

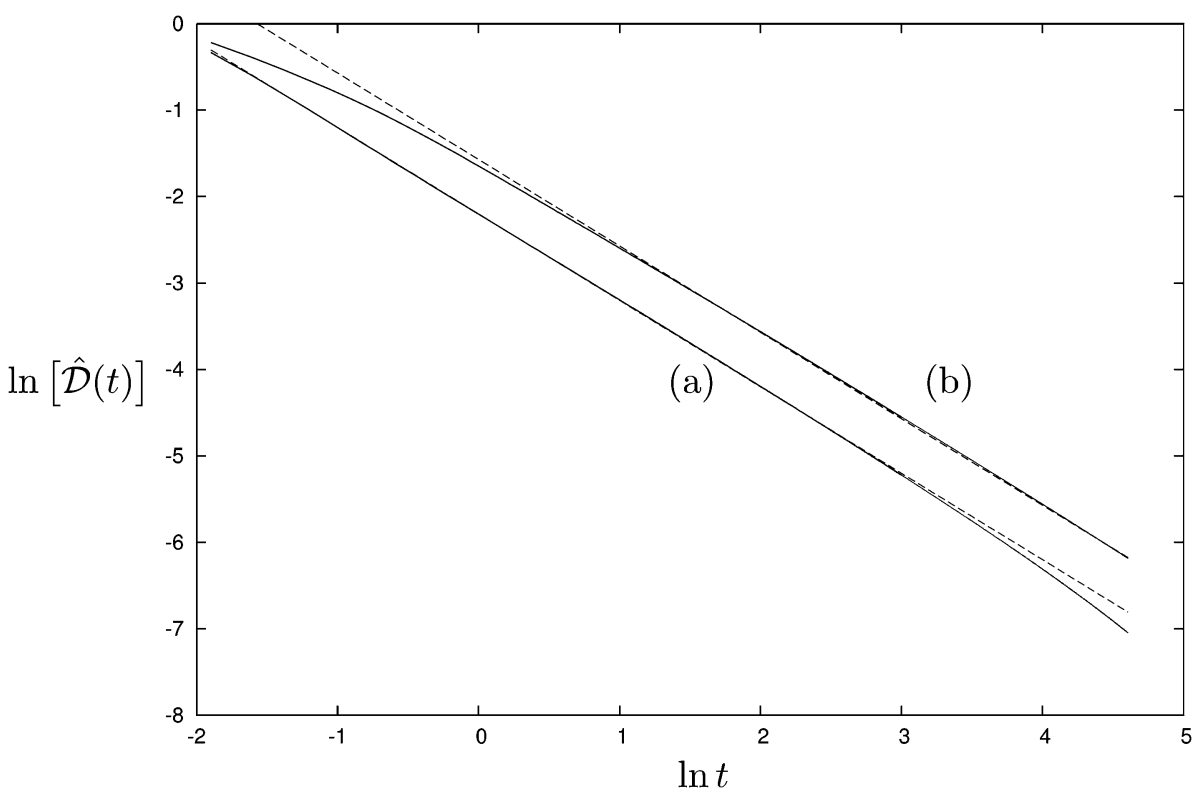

Fig. 6. The normalised deviation parameter $\ln \left[\widehat{\mathcal{D}}_{F}(t)\right]$ plotted as a function of $\ln t$ for currents evolving from the axisymmetric initial distributions (3.35) - (a) and (3.36) - (b). The graphs of the theoretically derived formulas (3.40) and (3.41) are also shown (dashed) for comparison.

convergence-see discussion below). This indicates that the $\theta$-dependence decays in time and the current approaches self-similar state.

Fig. 6 shows the logarithm of the normalised deviation parameter $\ln \left[\widehat{\mathcal{D}}_{F}(t)\right]$, where $\widehat{\mathcal{D}}_{F}(t)=\mathcal{D}_{F}(t) / \mathcal{D}_{F}\left(T_{0}\right)$, plotted as a function of $\ln t$, for the currents evolving from the axisymmetric initial profiles (3.35) and (3.36). The plots show that the value of $\widehat{\mathcal{D}}_{F}(t)$ decays in time as the currents adjust to self-similar state. For both currents, the $\ln \left[\widehat{\mathcal{D}}_{F}(t)\right]$ curves are shown to be essentially linear during the adjustment period, indicating a power law dependence of $\widehat{\mathcal{D}}_{F}(t)$ on time. Also plotted on the same graph are the theoretically derived formulas 


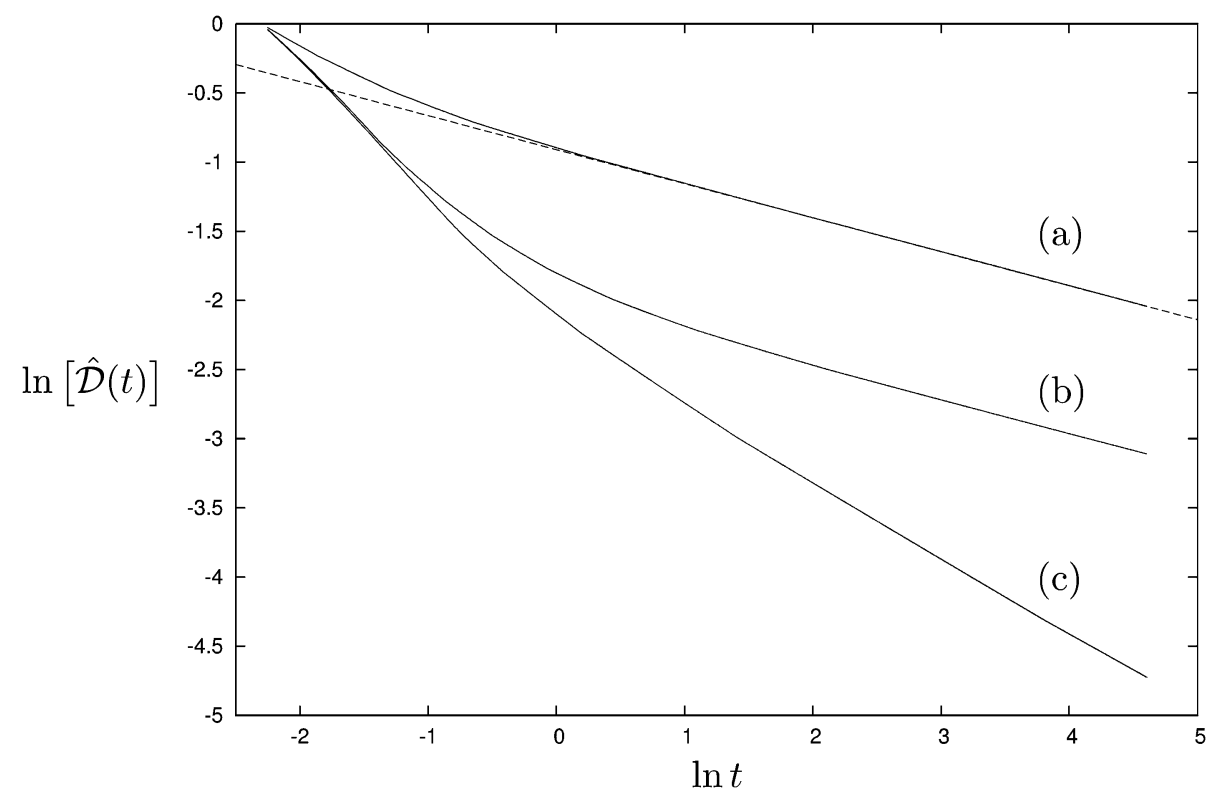

Fig. 7. The normalised deviation parameter $\ln \left[\widehat{\mathcal{D}}_{F}(t)\right]$ plotted as a function of $\ln t$ for currents evolving from the asymmetric initial distributions (3.37) - (a), (3.38) - (b), and (3.39) - (c). The empirically determined straight line $\ln \left[\widehat{\mathcal{D}}_{F}(t)\right]=-0.25 \ln t-0.9$ (dashed) is superimposed onto curve (a), demonstrating that $\widehat{\mathcal{D}}_{F}(t)$ decays like $t^{-1 / 4}$.

$$
\begin{aligned}
& \ln \left[\widehat{\mathcal{D}}_{F}(t)\right]=-\ln t-2.20, \\
& \ln \left[\widehat{\mathcal{D}}_{F}(t)\right]=-\ln t-1.57,
\end{aligned}
$$

describing the evolution of $\widehat{\mathcal{D}}_{F}(t)$ for the currents starting from (3.35) and (3.36), respectively (details of the derivations of these formulas are presented in Appendix B). The agreement between the numerical and theoretical results in the power-law regime is shown to be excellent, demonstrating that the linear theory not only predicts the correct power-law dependence of deviations on time, but also yields coefficients that agree with numerically measured values. Hence, these results confirm the theoretical assertion that volume-preserving, axisymmetric perturbations decay like $t^{-1}$ to leading order in terms of non-optimal variables.

Fig. 7 shows the graphs of $\ln \left[\widehat{\mathcal{D}}_{F}(t)\right]$ for the currents evolving from the asymmetric distributions (3.37)-(3.39). Again the curves are shown to develop into linear functions of $\ln t$ after sufficiently long times. For the current starting from (3.37), the linear part of the curve is empirically found to be $\ln \left[\widehat{\mathcal{D}}_{F}(t)\right]=-0.25 \ln t-0.9$, implying a rate of convergence to the self-similar solution of $t^{-1 / 4}$. This is also in agreement with the theoretical predictions which estimated that volume-preserving, $\theta$-dependent perturbations to the $\mathrm{B}-\mathrm{P}$ solution (in terms of non-optimal variables) will decay like $t^{-1 / 4}$ to leading order.

The graphs show that for the currents evolving from (3.38) and (3.39), the parameter $\widehat{\mathcal{D}}_{F}(t)$ initially decays like $t^{-1}$. Then, at large times, the rate of decay is shown to be $t^{-1 / 4}$ for the current evolving from (3.38) and $t^{-1 / 2}$ for the current evolving from (3.39). This behaviour can be explained as follows: for both profiles, the magnitude of the $\theta$-dependent term is significantly smaller than that of the axisymmetric term, and this is what makes (3.38) different from (3.37). Thus, the initial development of $\widehat{\mathcal{D}}_{F}(t)$ is determined by the axisymmetric component of the profile whose leading order rate of convergence to self-similar state is $t^{-1}$. Then, at large times, the rate of decay of $\widehat{\mathcal{D}}_{F}(t)$ is determined by the $\theta$-dependent terms. Hence the long-term rate of decay becomes $t^{-1 / 4}$ for the current evolving from (3.38). For the current evolving from (3.39), the long-term rate of decay is $t^{-1 / 2}$ because the eigenfunction expansion of the $\theta$-dependent component will involve only terms with $m=2$, for which the leading order term is characterised by $\lambda_{20}=1 / 2$ and decays like $t^{-1 / 2}$.

The foregoing numerical results were obtained by integrating the general initial-value problem (IVP) of the PME. We note that we have also performed numerical integrations starting from perturbed B-P profiles, and the results were essentially the same as above. This is to be expected because the perturbed B-P initial profiles represent a special case of the general IVP. 
These results confirm that currents evolving from arbitrary initial conditions that are not necessarily axisymmetric, approach the axisymmetric B-P solution in the intermediate regime. The rate of convergence to self-similar state has been shown to be faster for currents that evolve from axisymmetric initial profiles than those that evolve from asymmetric profiles in general. The numerical results confirm the theoretical predictions derived from the analysis of the two previous Sections.

\section{Concluding remarks}

We have revisited the problem of the linear stability of the axisymmetric Barenblatt-Pattle self-similar solution which was first studied by GM. We have reformulated the problem by transforming the dependent variable into a system where the slope of the self-similar height profile at the front remains finite for all values of $\alpha$. This approach eliminates singularities in the linear stability analysis which arise as a result of infinite gradients at the front of the height profile. We have extended the analysis to the case where the perturbations imposed depend of both the radial and azimuthal coordinates, and have established that the axisymmetric B-P solution is linearly stable to asymmetric disturbances. This is an important analytical result which represents a significant step towards showing that the B-P solution provides the intermediate asymptotics of currents that evolve from a wide range of initial conditions including those that are not axisymmetric.

The linear stability analysis has revealed that, for volume-preserving perturbations, axisymmetric and asymmetric perturbations decay like $t^{-1}$ and $t^{-1 /(2 \alpha+2)}$, respectively, to leading order. A consideration of the connection between the perturbation eigenfunctions and the symmetry transformations of the B-P solution has shown that the first two axisymmetric eigenfunctions $\left(\mathcal{F}_{00}\right.$ and $\left.\mathcal{F}_{01}\right)$ and the first asymmetric eigenfunction $\left(\mathcal{F}_{10}\right)$ are equivalent to the coefficients of the infinitesimal volume-altering, time-shifting and space-translating transformations, respectively. This means that, for a given perturbation function, it is always possible to redefine the volume, time and space variables so that $\mathcal{F}_{00}=\mathcal{F}_{01}=\mathcal{F}_{10}=0$. In terms of these optimal variables, therefore, axisymmetric and asymmetric perturbation decay like $t^{-4+2 /(\alpha+1)}$ and $t^{-1 /(\alpha+1)}$, respectively, to leading order. Thus, both in terms of primitive and optimal variables, axisymmetric perturbations decay faster than asymmetric ones implying that currents evolving from axisymmetric initial distributions will typically adjust to self-similar state more rapidly than those evolving from $\theta$-dependent distributions.

We have calculated numerical solutions for the development of currents evolving from arbitrary axisymmetric and asymmetric distributions. The results confirm that currents evolving from both axisymmetric and asymmetric initial distributions approach the axisymmetric B-P solution at large times. The rates of adjustment to self-similar state have been found to be faster for currents with axisymmetric initial profiles compared to those of currents with asymmetric initial profiles. Even though the initial distributions were arbitrary functions different from weakly perturbed B-P profiles, the numerically determined rates of adjustment were found to be in excellent agreement with those predicted by the linear theory.

\section{Acknowledgements}

The authors would like to thank two anonymous referees, from whose comments the paper has greatly benefited. JSM gratefully acknowledges the financial support of the Commonwealth Scholarship Commission (UK).

\section{Appendix A. Numerical methods}

The governing equation (3.31) (with $\alpha=1$ ) was integrated numerically on an $R-\theta$ grid, representing the semicircular region $0 \leqslant R \leqslant 1,0 \leqslant \theta \leqslant \pi$, using an explicit forward-time, centred-space finite difference scheme (Press et al. [31])

$$
\mathcal{H}_{i, j}^{n+1}=\mathcal{H}_{i, j}^{n}+\frac{\Delta t}{r_{F j}} R_{i} \dot{r}_{F j} \partial_{R}\left(\mathcal{H}_{i, j}^{n}\right)+\Delta t \frac{1}{r_{F j}^{2}} \nabla_{R \theta}^{2}\left[\frac{1}{2}\left(\mathcal{H}_{i, j}^{n}\right)^{2}\right],
$$


where $\mathcal{H}_{i, j}^{n}=\mathcal{H}\left(R_{i}, \theta_{j}, t\right)=\mathcal{H}(i \Delta R, j \Delta \theta, n \Delta t)$. The values of $r_{F j}$ were updated at each time-step using the following formula:

$$
\dot{r}_{F j}=\frac{1}{r_{F j}}\left(1+\frac{r_{F}^{\prime 2}}{r_{F j}^{2}}\right)\left[\partial_{R} \mathcal{H}\right]_{R=1} .
$$

The scheme (A.1) is stable provided $\Delta t \leqslant \frac{1}{2} \mathcal{H}^{-1}\left(1 /(\Delta R)^{2}+1 /(\Delta \theta)^{2}\right)^{-1}$ (Morton and Mayers [32]). It is secondorder in space and first-order in time. For example, when $\Delta R=0.04$ and $\Delta \theta=\frac{1}{20} \pi$, the time step of $\Delta t=0.00001$ was found to be sufficiently small to maintain numerical stability. All integrations were performed from the initial time $T_{0}=0.1$.

Near $R=0$. In the neighbourhood of $R=0$, we expand $\mathcal{H}$ in a Taylor series keeping $\theta$ and $t$ fixed:

$$
\mathcal{H}(i \Delta R, \theta, t)=\mathcal{H}_{i}=\mathcal{G}_{0}(t)+\mathrm{i} \Delta R \mathcal{G}_{1}(\theta, t)+\frac{1}{2}(\mathrm{i} \Delta R)^{2} \mathcal{G}_{2}(\theta, t)+\cdots,
$$

where $\mathcal{G}_{n}=\left[\partial^{n} \mathcal{H} / \partial R^{n}\right]_{R=0}$. An $\mathrm{O}\left((\Delta R)^{2}\right)$ estimate of $\mathcal{H}_{0}$ in terms of $\mathcal{H}_{1}, \mathcal{H}_{2}$ and $\mathcal{H}_{3}$ is then given by

$$
\mathcal{H}_{0}=3\left(\mathcal{H}_{1}-\mathcal{H}_{2}\right)+\mathcal{H}_{3}
$$

This expression was used to update $\mathcal{H}_{0}$ at each time-step and it is equivalent to requiring that the curvature of $\mathcal{H}$ at the origin be equal to that at the neighbouring points.

The results of the numerical calculations were validated against the B-P self-similar solution. Integrations from the B-P solution produced profiles that remained self-similar at subsequent times and conserved volume to a high degree of accuracy. For example, when $\Delta R=0.01$, volume was conserved to within $99.99 \%$ throughout the computation.

\section{Appendix B. Theoretical derivation of expressions for $\ln \left[\widehat{\mathcal{D}}_{F}(t)\right]$}

We present theoretically derived formulas which describe the evolution of the deviation parameter $\widehat{\mathcal{D}}_{F}(t)$ for currents that start from the initial profiles (3.35) and (3.37), respectively.

Consider first the initial profile (3.35)

$$
\mathcal{H}_{P 1}(r)=\sqrt{\frac{5}{2}}[1-\beta r]_{+} .
$$

We assume that this profile, with volume $V=\int_{0}^{r_{F}} r \mathcal{H} \mathrm{d} r=\frac{2}{3}$, is produced by a volume-preserving perturbation of a B-P similarity solution $\mathcal{H}_{S}$, at time $t=t_{0}$. We choose $t=t_{0}$ to be the time at which the front of the unperturbed profile $\mathcal{H}_{S}$ matches that of $\mathcal{H}_{P 1}$, that is

$$
r_{F}=\left(32 V t_{0}\right)^{1 / 4}=1 / \beta \text {. }
$$

This gives $t_{0}=\frac{3}{10}$. Then, writing $\mathcal{H}_{P 1}=t_{0}^{-1 / 2} \Psi_{P 1}(\eta)$ and $\mathcal{H}_{S}=t_{0}^{-1 / 2} \Psi_{S}(\eta)$, yields

$$
\begin{aligned}
& \Psi_{P 1}(\eta)=\frac{1}{2} \sqrt{3}(1-\eta)_{+}, \\
& \Psi_{S}(\eta)=\frac{1}{3} \sqrt{3}\left(1-\eta^{2}\right)_{+},
\end{aligned}
$$

where $\eta=r / r_{F}$. At $t=t_{0}$, we have

$$
\Psi_{P 1}(\eta)=\Psi_{S}(\eta)+\sum_{n=0}^{\infty} a_{n} P_{n}\left(2 \eta^{2}-1\right)
$$

from which the first four coefficients are found to be $a_{0}=0, a_{1}=-\frac{1}{30} \sqrt{3}, a_{2}=\frac{1}{30} \sqrt{3}$ and $a_{3}=-\frac{1}{45} \sqrt{3}$.

The numerical calculations were started at $t=T_{0}=\frac{1}{10}$ so that $t=T_{0}+T$ at subsequent times. The deviation parameter can be written as

$$
\mathcal{D}_{F}(t)=\left|1-\frac{r_{F}}{(32 V t)^{1 / 4}}\right|=\left|1-\left(\frac{t_{0}+T}{T_{0}+T}\right)^{1 / 4} \eta_{F}\left(t_{0}+T\right)\right|=\left|1-\left(\frac{t_{0}+T}{T_{0}+T}\right)^{1 / 4}\left(1+\eta_{F 1}\left(t_{0}+T\right)\right)\right| .
$$


Expanding for $T \gg 1$ and using (3.26) then yields

$$
\widehat{\mathcal{D}}_{F}(t)=\frac{\mathcal{D}_{F}(t)}{\mathcal{D}_{F}\left(T_{0}\right)} \sim \frac{\left|\left(T_{0}-t_{0}\right)-16 a_{1} t_{0} / \xi_{F 0}^{2}\right|}{4\left|1-\left(t_{0} / T_{0}\right)^{1 / 4}\right|} \frac{1}{T},
$$

to leading order.

For the initial profile $h_{P 1}$, with $T_{0}=\frac{1}{10}, t_{0}=\frac{3}{10}, \xi_{F 0}^{4}=\frac{64}{3}$ and $a_{1}=-\frac{1}{30} \sqrt{3}$, the formula (B.5) gives

$$
\ln \left[\widehat{\mathcal{D}}_{F}(t)\right] \sim-\ln T-2.20 \text {. }
$$

Similar calculations for the initial profile (3.36)

$$
\mathcal{H}(r)=\sqrt{\frac{5}{2}} \begin{cases}\left(5-5^{4 / 5}\right) \beta r, & 0 \leqslant \beta r<\frac{1}{5}, \\ 1-(\beta r)^{1 / 5}, & \frac{1}{5} \leqslant \beta r \leqslant 1,\end{cases}
$$

give $T_{0}=\frac{1}{10}, V=0.1692, t_{0}=1 /(5 V), \xi_{F 0}^{4}=32 V$ and $a_{1}=-0.046$. The evolution equation for $\widehat{\mathcal{D}}_{F}$ is thus found to be

$$
\ln \left[\widehat{\mathcal{D}}_{F}(t)\right] \sim-\ln T-1.57
$$

The expressions (B.6) and (B.7) have been shown to be in excellent agreement with numerical results.

\section{References}

[1] J. Bear, Dynamics of Fluids in Porous Media, Dover, 1988.

[2] F.A.L. Dullien, Porous Media: Fluid Transport and Pore Structure, Academic, 1992.

[3] H.E. Huppert, A.W. Woods, Gravity-driven flows in porous layers, J. Fluid Mech. 292 (1995) 55-69.

[4] G.I. Barenblatt, Scaling, Self-Similarity, and Intermediate Asymptotics, Cambridge University Press, 1996.

[5] A.C. Fowler, Mathematical Models in the Applied Sciences, Cambridge University Press, 1997.

[6] G.I. Barenblatt, On some unsteady motions of fluids and gases in a porous medium, Prik. Mat. Mekh. 16 (1952) $67-78$.

[7] Ya.B. Zel'dovich, A.S. Kompaneets, On the theory of propagation of heat with thermal conductivity depending on temperature, in: Collection of Papers Dedicated to the 70th Birthday of A.F. Ioffe, Izd. Akad. Nauk USSR, Moscow, 1950, pp. 61-71.

[8] R.E. Pattle, Diffusion from an instantaneous point source with a concentration dependent coefficient, Quart. J. Mech. Appl. Math. 12 (1959) 407-409.

[9] H.E. Huppert, The propagation of two-dimensional and axisymmetric viscous gravity currents over a rigid horizontal surface, J. Fluid Mech. 121 (1982) 43-58.

[10] R.E. Grundy, R. McLaughlin, Eigenvalues of the Barenblatt-Pattle similarity solution in nonlinear diffusion, Proc. Roy. Soc. London Ser. A 383 (1982) 89-100.

[11] T.P. Witelski, A.J. Bernoff, Self-similar asymptotics for linear and nonlinear diffusion equations, St. Appl. Math. 100 (1998) $153-193$.

[12] J.R. King, Exact multidimensional solutions to some nonlinear diffusion equations, Quart. J. Mech. Appl. Math. 46 (1993) $419-436$.

[13] P. Halfar, On the dynamics of the Ice Sheets, J. Geophys. Res. 86 (1981) 11065-11072.

[14] P. Halfar, On the dynamics of the Ice Sheets 2, J. Geophys. Res. 88 (1983) 6043-6051.

[15] A.J. Bernoff, T. P Witelski, Linear stability of source-type similarity solutions of the thin film equation, Appl. Math. Lett. 15 (2002) 599-606.

[16] A. Chertock, On the stability of a class of self-similar solutions to the filtration-absorption equation, Eur. J. Appl. Math. 13 (2002) 179-194.

[17] D. Pritchard, A.W. Woods, A.J. Hogg, On the slow draining of a gravity current moving through a layered permeable medium, J. Fluid Mech. 444 (2001) 23-47.

[18] J. Boussinesq, Recherches théoriques sur l'écoulement des nappes d'eau infiltres dans le sot et sur débit de sources, C. R. H. Acad. Sci., J. Math. Pures Appl. 10 (1903) 6-78, 363-394.

[19] M. Muskat, The Flow of Homogeneous Fluids through Porous Media, McGraw-Hill, New York, 1937.

[20] Ya.B. Zel'dovich, Yu.P. Raizer, Physics of Shock Waves and High Temperature Hydrodynamic Phenomena, vol. I, Academic Press, New York, 1966.

[21] E.W. Larsen, G.C. Pomraning, Asymptotic analysis of waves, SIAM J. Appl. Math. 39 (1980) 201-212.

[22] J.D. Murray, Mathematical Biology, Springer-Verlag, London, 1989.

[23] T.P. Witelski, Segregation and mixing in degenerate diffusion in population dynamics, J. Math. Biol. 35 (1997) 695-712.

[24] L.A. Peletier, The porous media equation, in: H. Amman, N. Bazley, K. Kirchgässner (Eds.), Applications of Nonlinear Analysis in the Physical Sciences, in: Surveys and Reference Works in Mathematics, Pitman, Boston, London, 1981.

[25] D.G. Aronson, The porous medium equation, in: A. Fasano, M. Primicerio (Eds.), Nonlinear Diffusion Problems, in: Lecture Notes in Math., vol. 1224, Springer-Verlag, New York, 1986.

[26] J. Gilchrist, Flux diffusion and the porous medium equation, Physica C 291 (1997) 132-142.

[27] M. Abramowitz, I.A. Stegun, Handbook of Mathematical Functions, Dover Publications Inc., 1965.

[28] L. Dresner, Applications of Lie's Theory of Ordinary and Partial Differential Equations, Institute of Physics Publishing, 1999. 
[29] M.E. Gurtin, R.C. MacCamy, E.A. Socolovsky, A coordinate transformation for the porous medium equation that renders the free-boundary stationary, Quart. Appl. Math. 42 (1984) 345-357.

[30] J.G. Berryman, Evolution of a stable profile for a class of nonlinear diffusion equations. III. Slow diffusion on the line, J. Math. Phys. 21 (6) (1980) 1326-1331.

[31] W.H. Press, S.A. Teukolsky, W.T. Vetterling, B.P. Flannery, Numerical Recipes in ForTRAN, Cambridge University Press, 1992.

[32] K.W. Morton, D.F. Mayers, Numerical Solutions of Partial Differential Equations, Cambridge University Press, 1994. 